\title{
Emissão de óxidos de nitrogênio associada à aplicação de uréia sob plantio convencional e direto
}

\author{
Arminda Moreira de Carvalho(1), Mercedes Maria da Cunha Bustamante ${ }^{(2)}$, Alessandra Rodrigues Kozovits ${ }^{(2)}$, \\ Leo Nobre de Miranda ${ }^{(1)}$, Lúcio José Vivaldi(2) e Danielle Matias de Sousa ${ }^{(2)}$
}

(1)Embrapa Cerrados, Rod. BR 020, Km 18, CEP 73301-970 Planaltina, DF. E-mail: arminda@cpac.embrapa.br, leo@cpac.embrapa.br

(2)Universidade de Brasília, Dep. de Ecologia, CEP 70919-970 Brasília, DF. E-mail: mercedes@unb.br, kozovits@unb.br, vivaldi@unb.br

\begin{abstract}
Resumo - O objetivo deste trabalho foi avaliar emissões de $\mathrm{NO}$ e $\mathrm{N}_{2} \mathrm{O}$ até cinco dias após a primeira fertilização de cobertura com uréia em milho, em Latossolo Vermelho argiloso distrófico, sob plantio convencional e direto. A adubação de cobertura foi de $60 \mathrm{~kg} \mathrm{ha}^{-1} \mathrm{de}$ N. O experimento foi conduzido na Embrapa Cerrados, Planaltina, $\mathrm{DF}$, com delineamento de blocos ao acaso, com três repetições, sendo o terceiro cultivo de milho, em rotação com soja. Os fluxos de $\mathrm{NO}$ e $\mathrm{N}_{2} \mathrm{O}$ foram medidos em câmaras de PVC instaladas em cada parcela. Houve emissão alta de NO imediatamente após $\left(5,4 \mathrm{ng} \mathrm{N} \mathrm{cm}^{-2} \mathrm{~h}^{-1}\right)$ e no terceiro dia $\left(4,8 \mathrm{ng} \mathrm{N} \mathrm{cm}^{-2} \mathrm{~h}^{-1}\right)$ após aplicação de uréia e irrigação. Um dia após fertilização, a emissão de NO reduziu-se a $1,9 \mathrm{ng} \mathrm{N} \mathrm{cm}^{-2} \mathrm{~h}^{-1}$, e cinco dias depois, alcançou $1,2 \mathrm{ng} \mathrm{N} \mathrm{cm}{ }^{-2} \mathrm{~h}^{-1}$. Os fluxos de $\mathrm{N}_{2} \mathrm{O}$ ficaram abaixo do limite de detecção de $0,6 \mathrm{ng} \mathrm{N} \mathrm{cm}^{-2} \mathrm{~h}^{-1}$. Não houve diferença significativa entre os plantios convencional e direto quanto à emissão dos óxidos de nitrogênio.
\end{abstract}

Termos para indexação: óxido nítrico, óxido nitroso, fertilização nitrogenada, plantas de cobertura, irrigação, Cerrado.

\section{Nitrogen oxides emission related to urea broadcasting fertilization under conventional and no-tillage systems}

\begin{abstract}
The objective of this work was to evaluate the $\mathrm{NO}$ and $\mathrm{N}_{2} \mathrm{O}$ emissions up to five days after the first nitrogen broadcasting fertilization with urea in a cornfield, in a Red Latosol, under conventional and no-tillage systems. The level of nitrogen broadcasting fertilization was of $60 \mathrm{~kg} \mathrm{ha}^{-1}$. The experiment was conducted at Embrapa Cerrados, Planaltina, DF, Brazil, in a randomized block design, with three replications, beeing the third corn crop in rotation with soybean. $\mathrm{NO}$ and $\mathrm{N}_{2} \mathrm{O}$ fluxes were measured using PVC chambers installed in each plot. Higher NO fluxes were found immediately after $\left(5.4 \mathrm{ng} \mathrm{N} \mathrm{cm}^{-2} \mathrm{~h}^{-1}\right)$ and three days $\left(4.8 \mathrm{ng} \mathrm{N} \mathrm{cm}^{-2} \mathrm{~h}^{-1}\right)$ after $\mathrm{N}$ fertilization and irrigation. In the first day after fertilization, the $\mathrm{NO}$ emission decreased significantly to $1.9 \mathrm{ng} \mathrm{N} \mathrm{cm}^{-2} \mathrm{~h}^{-1}$, and after five days, reached $1.2 \mathrm{ng} \mathrm{N} \mathrm{cm}^{-2} \mathrm{~h}^{-1}$. $\mathrm{N}_{2} \mathrm{O}$ fluxes values were below the detection limit of $0.6 \mathrm{ng} \mathrm{N} \mathrm{cm}^{-2} \mathrm{~h}^{-1}$. No significant differences in nitrogen oxides fluxes were found between plots under conventional and no-tillage systems.
\end{abstract}

Index terms: nitric oxide, nitrous oxide, nitrogen fertilization, cover crops, irrigation, Cerrado.

\section{Introdução}

Concentrações atmosféricas de gases traços como $\mathrm{NO}$ e $\mathrm{N}_{2} \mathrm{O}$ têm aumentado rapidamente devido a atividades antropogênicas - queima de combustíveis fósseis, urbanização, desmatamentos e queimadas - e em especial, às atividades agrícolas com utilização intensiva de fertilizantes, revolvimento do solo e incorporação de resíduos vegetais, irrigação, drenagem, entre outras (Sanhueza et al., 1994; Skiba et al., 1997).
Os óxidos de nitrogênio ( $\mathrm{NO}$ e $\mathrm{N}_{2} \mathrm{O}$ ) são produtos das reações de nitrificação e desnitrificação. A nitrificação produz relativamente mais $\mathrm{NO}$ e a desnitrificação é o processo dominante na produção do $\mathrm{N}_{2} \mathrm{O}$ (Davidson et al., 1993). A nitrificação é favorecida pela presença de $\mathrm{NH}_{4}{ }^{+}$, por condições adequadas de aeração do solo e pela maior ciclagem de nitrogênio no sistema. A magnitude da emissão de NO do solo depende das taxas de nitrificação, desnitrificação e das propriedades de difusão do solo (Skiba et al., 1997; Davidson et al., 2000). O nitrato, por sua vez, pode se 
acumular no solo quando produzido além da demanda de microrganismos e plantas, potencializando reações de desnitrificação (Matson et al., 1999).

As emissões de $\mathrm{NO}$ e $\mathrm{N}_{2} \mathrm{O}$ em solos agrícolas estão fortemente associadas à fertilização nitrogenada. Embora esta seja uma prática comum nos sistemas de cultivos intensivos e mecanizados do Cerrado, poucos trabalhos têm avaliado as emissões de $\mathrm{N}_{2} \mathrm{O}$ em áreas de culturas anuais nesta região (Nobre, 1994; Saminêz, 1999). Não existem relatos de emissões de NO em áreas agrícolas de Cerrado.

A substância aplicada (uréia, nitrato ou amônio) e o modo de aplicação dos fertilizantes nitrogenados (na superfície ou incorporado, na linha de semeadura ou nas entre linhas) influenciam o fluxo de NO (Sanhueza et al., 1994; Davidson et al., 1996; Skiba et al., 1997). Slemr \& Seiler (1991) e Paul et al. (1993) relacionaram o incremento do fluxo de NO com o tipo de fertilizante aplicado e com o $\mathrm{NH}_{4}{ }^{+}$disponível no solo. Vos et al. (1994) observaram que o fluxo de NO foi mais elevado ao se incorporar adubos verdes, comparativamente ao pousio, e também verificaram relação positiva entre o fluxo de $\mathrm{NO}$ e a concentração de $\mathrm{NH}_{4}{ }^{+}$no solo. As maiores emissões de $\mathrm{N}_{2} \mathrm{O}$ medidas em cultivos de cana-de-açúcar também se relacionaram positivamente à concentração de $\mathrm{NH}_{4}{ }^{+}$no solo (Davidson et al., 1996).

A umidade e aeração do solo, representadas pela porcentagem de espaços dos poros preenchidos pela água (EPPA) e a temperatura, contribuem para potencializar ou não o efeito da aplicação de nitrogênio (Vos et al., 1994; Davidson et al., 2000). A umidade do solo, a forma (substância) e o modo de aplicação do adubo geralmente determinam a duração das altas emissões (picos) dos óxidos de nitrogênio após a fertilização (Davidson et al., 1996; Skiba et al., 1997; Skiba et al., 1998; Veldkamp et al., 1998). O uso de leguminosas fixadoras de $\mathrm{N}_{2}$ nos sistemas agrícolas também pode aumentar os teores deste nutriente no solo e as emissões de $\mathrm{NO}$ e $\mathrm{N}_{2} \mathrm{O}$. O fluxo de $\mathrm{N}_{2} \mathrm{O}$ medido em cultura de soja, no Cerrado, na estação chuvosa, foi mais elevado do que os observados em pastagem e cerrado natural (Saminêz, 1999).

A dinâmica dos fluxos dos óxidos de nitrogênio pode ser afetada ainda pelo sistema de preparo do solo (convencional ou plantio direto). Civerolo \& Dickerson (1998) sugeriram que as menores emissões de $\mathrm{NO}$ em solo cultivado com milho sob plantio direto ocorreram em virtude da menor exposição do solo e da maior proteção por plantas de cobertura. O incremento do fluxo de NO após o preparo de solo tem sido atribuído ao aumento de $\mathrm{NO}_{3}{ }^{-}$, às mudanças nas taxas de movimentação no solo pelas alterações na porosidade e à exposição da superfície do solo diretamente em contato com a atmosfera (Cárdenas et al., 1993; Sanhueza et al., 1994).

Embora solos sob vegetação nativa de Cerrado e sob pastagem apresentem fluxos baixos de $\mathrm{NO}$ e $\mathrm{N}_{2} \mathrm{O}$ (Pinto et al., 2002; Varella et al., 2004), alta emissão de NO ocorre depois de queimadas ou após chuvas em seqüência a períodos secos (Pinto et al., 2002; Varella et al., 2004). Esses fluxos elevados, mesmo que de curta duração, podem ser importantes no balanço anual da emissão de gases.

No caso da uréia aplicada na superfície do solo, a principal perda de nitrogênio ocorre por meio de volatilização na forma de gás $\mathrm{NH}_{3}$, podendo chegar a $70 \%$ do nitrogênio. A lixiviação também é um processo relevante de perdas de nitrogênio no Cerrado, principalmente em áreas de alta precipitação e solos bem drenados (Sousa \& Lobato, 2004).

O objetivo deste trabalho foi avaliar o efeito da aplicação de nitrogênio mineral a uma cultura de milho em sucessão a Mucuna aterrima (leguminosa fixadora de $\mathrm{N}_{2}$ ) sobre as emissões de $\mathrm{NO}$ e $\mathrm{N}_{2} \mathrm{O}$, em solo de Cerrado, sob plantio convencional e direto.

\section{Material e Métodos}

O trabalho foi conduzido na Embrapa Cerrados, Planaltina, DF. O clima é classificado como Tropical Estacional (Aw), conforme Köppen, e o solo como Latossolo Vermelho argiloso distrófico. Foram determinadas as características granulométricas e químicas do solo, na profundidade de $0-20 \mathrm{~cm}$, antes da implantação do experimento (2000) e antes da semeadura do milho (2003) (Tabela 1).

Em novembro de 2000, iniciou-se o cultivo de soja, seguida de milho nas estações chuvosas, em plantio direto (sem preparo do solo) e plantio convencional do solo (aração e gradagem). As plantas de cobertura, mucuna-preta (Mucuna aterrima) e milheto cv. BR 05 (Pennisetum glaucum L.) foram intercaladas no período seco, estabelecendo-se o sistema de sucessão soja seguida de mucuna, milho e milheto. As parcelas receberam 4 t ha $^{-1}$ de calcário (PRNT $\left.=100 \%\right)$, antes da implantação do sistema plantio direto e preparo convencional do solo (início do experimento), para elevar a saturação por bases do solo a 50\%. O delineamento experimental foi o de blocos ao acaso com três repetições, em parcelas de 8x15 m. 
As medidas dos fluxos de $\mathrm{NO}$ e $\mathrm{N}_{2} \mathrm{O}$ foram efetuadas no solo sob cultura do milho em plantio direto e plantio convencional, no ano agrícola 2003/2004. Na semeadura da cultura (10 de novembro de 2003) foi realizada adubação de manutenção no sulco de plantio, utilizando $500 \mathrm{~kg} \mathrm{ha}^{-1}$ da fórmula NPK 4-30-16 (20 kg ha- ${ }^{-1}$ de N na forma de uréia) acrescida de $2 \mathrm{~kg} \mathrm{ha}^{-1}$ de Zn $\left(\mathrm{ZnSO}_{4} .7 \mathrm{H}_{2} \mathrm{O}\right)$. O milho recebeu posteriormente duas aplicações de nitrogênio em cobertura, em 10 e 20 de dezembro de 2003, sendo cada uma de $60 \mathrm{~kg} \mathrm{ha}^{-1}$ de $\mathrm{N}$ na forma de uréia. A uréia foi aplicada na superfície do solo e ao lado do sulco de plantio. Em seguida, a área recebeu uma lâmina aproximada de $25 \mathrm{~mm}$ de água, por aspersão. A precipitação pluvial acumulada, nos dez dias anteriores à aplicação de uréia, foi de $41 \mathrm{~mm}$.

A primeira avaliação dos fluxos de $\mathrm{NO}$ e de $\mathrm{N}_{2} \mathrm{O}$ foi realizada em 10 de dezembro de 2003, ou seja, trinta dias após a semeadura do milho, com a coleta iniciando-se 15 minutos após a aplicação da uréia (primeira fertilização em cobertura), ao lado da linha de semeadura. As demais medidas dos fluxos de NO foram efetuadas um dia, três dias e cinco dias após a aplicação da uréia. As coletas de $\mathrm{N}_{2} \mathrm{O}$ também iniciaram-se 15 minutos após a aplicação da uréia, seguindo depois de um, dois, três e cinco dias desta fertilização em cobertura.

As medidas foram realizadas uma vez ao dia, nos três blocos do experimento, sempre no período das 10-14 horas, com a finalidade de reduzir a variação das medidas dos fluxos de gases. Quatro suportes de PVC foram colocados, aleatoriamente, em cada tratamento, no mínimo trinta minutos antes do início das coletas. Os suportes foram inseridos ao lado da linha de semeadura, de modo a coletar os gases de nitrogênio (NO e $\mathrm{N}_{2} \mathrm{O}$ ) onde se aplicou uréia. Câmaras de PVC de 20,0 cm de altura por 24,2 cm de diâmetro foram acopladas aos anéis para as medidas. O volume total do conjunto câmara-anel, para as coletas de $\mathrm{NO}$ e de $\mathrm{N}_{2} \mathrm{O}$, foi de $7,0 \mathrm{~L}$.

Tabela 1. Caracterização granulométrica e química $(0-20 \mathrm{~cm})$ de um Latossolo Vermelho argiloso distrófico, antes da implantação do experimento (AI), em 2000, e antes da semeadura do milho (AS), em 2003.

\begin{tabular}{|c|c|c|}
\hline Características do solo & $\mathrm{AI}$ & $\mathrm{AS}$ \\
\hline $\operatorname{Argila}\left(\mathrm{g} \mathrm{kg}^{-1}\right)$ & 590 & - \\
\hline Silte $\left(\mathrm{g} \mathrm{kg}^{-1}\right)$ & 80 & - \\
\hline $\operatorname{Areia}\left(\mathrm{g} \mathrm{kg}^{-1}\right)$ & 330 & - \\
\hline $\mathrm{pH}$ em água $(1: 2,5)$ & 5,1 & 5,5 \\
\hline Densidade do solo $\left(\mathrm{g} \mathrm{cm}^{-3}\right)$ & - & 1,1 \\
\hline $\mathrm{C}\left(\mathrm{g} \mathrm{kg}^{-1}\right)$ & 27 & - \\
\hline $\mathrm{Al}^{3+}\left(\mathrm{cmol}_{\mathrm{c}} \mathrm{dm}^{-3}\right)$ & 0,93 & 0,10 \\
\hline $\mathrm{H}+\mathrm{Al}\left(\mathrm{cmol}_{\mathrm{c}} \mathrm{dm}^{-3}\right)$ & 5,8 & 4,9 \\
\hline $\mathrm{K}^{+}\left(\mathrm{cmol}_{\mathrm{c}} \mathrm{dm}^{-3}\right)$ & 0,04 & 0,16 \\
\hline $\mathrm{Ca}^{2+}+\mathrm{Mg}^{2+}\left(\mathrm{cmol}_{\mathrm{c}} \mathrm{dm}^{-3}\right)$ & 0,18 & 2,60 \\
\hline Saturação por bases ou V (\%) & 3,7 & 36,0 \\
\hline $\mathrm{P}_{\text {Mehlich-1 }}\left(\mathrm{mg} \mathrm{dm}^{-3}\right)$ & 1,2 & 9,3 \\
\hline
\end{tabular}

O método da câmara dinâmica foi utilizado para determinar o fluxo de NO, com um analisador de quimiluminescência (NoxBox) associado ao datalogger. O conjunto câmara-anel foi conectado ao NoxBox, que permaneceu fechado durante cinco minutos, para a leitura em cada base. O fluxo de NO do solo foi calculado com base na porção linear da relação entre o tempo e concentração dentro da câmara, com o mínimo de um minuto de leitura, após o acoplamento da câmara. Como o detector de $\mathrm{NO}_{2}$ deste aparelho é sensível a baixas concentrações, utilizou-se uma equação apropriada a este tipo de resposta: $\mathrm{Y}=\mathrm{aX}+\mathrm{bX} \mathrm{X}^{0,5}+\mathrm{c}$, em que $\mathrm{Y}$ é a concentração de NO e X é o tempo, em minutos. O limite de detecção determinado para NO, por Verchot et al. (1999), num sistema idêntico ao deste trabalho, foi de $0,01 \mathrm{ng} \mathrm{cm}^{-2} \mathrm{~h}^{-1}$.

Os fluxos de $\mathrm{N}_{2} \mathrm{O}$ foram determinados pelo método da câmara estática. Depois do acoplamento da câmara ao anel (tempo zero), três amostras de ar (10, 20 e 30 minutos), de $40 \mathrm{~mL}$, foram coletadas com seringas de náilon ( $60 \mathrm{~mL}$ ) adaptadas com válvula de segurança, que impediram trocas gasosas entre o meio externo e interno das seringas. As amostras foram analisadas no mesmo dia da coleta, a fim de minimizar a perda potencial de $\mathrm{N}_{2} \mathrm{O}$ das seringas. A concentração de $\mathrm{N}_{2} \mathrm{O}$ nas amostras foi determinada por meio de cromatografia gasosa em um cromatógrafo Shimadzu GC-14A equipado com detector de captura de elétrons $\left({ }^{63} \mathrm{Ni}\right)$. As análises foram efetuadas com o software Peaksimple. Os fluxos de $\mathrm{N}_{2} \mathrm{O}$ foram calculados por meio da regressão linear da concentração do gás pelo tempo. Adotou-se o limite de detecção para $\mathrm{N}_{2} \mathrm{O}$ de $0,6 \mathrm{ng} \mathrm{cm}^{-2} \mathrm{~h}^{-1}$, determinado por Verchot et al.(1999), que utilizaram os mesmos procedimentos de coleta e configuração do cromatógrafo idêntica à utilizada neste trabalho.

As temperaturas do ar e do solo (2,5 e 5,0 cm de profundidade) foram determinadas por termopares nas quatro bases, durante as avaliações dos fluxos dos óxidos de nitrogênio.

Quatro amostras de solo foram coletadas, em cada parcela, para determinação da concentração de nitrogênio inorgânico - N do $\mathrm{NO}_{3}{ }^{-}\left(\mathrm{N}^{-} \mathrm{NO}_{3}\right)^{-}$e $\mathrm{N}$ amoniacal $\left(\mathrm{N}_{-} \mathrm{NH}_{4}{ }^{+}\right)$- disponível no solo. As coletas foram efetuadas no interior dos anéis, na profundidade de $0-5 \mathrm{~cm}$, em cada uma das datas de medidas dos fluxos de óxidos de nitrogênio.

As amostras de solo foram mantidas em uma caixa refrigerada e transportadas ao laboratório para extração e determinação do nitrogênio inorgânico. Dois gramas de terra fresca foram agitados por uma hora com $25 \mathrm{~mL}$ de $\mathrm{KCl}$ (2,0 M), para extração do nitrogênio inorgânico. As leituras de $\mathrm{NO}_{3}{ }^{-}$foram realizadas pelo método de Meier (1991). $\mathrm{O} \mathrm{NH}_{4}{ }^{+}$foi determinado utilizando-se o método colorimétrico de Nessler (Greweling \& Peech, 1960). O limite inferior da curva de calibração para $\mathrm{NO}_{3}{ }^{-}$foi de $1,0 \mathrm{mg} \mathrm{kg}^{-1}$ e, para $\mathrm{NH}_{4}{ }^{+}$, 
de $2,5 \mathrm{mg} \mathrm{kg}^{-1}$, sendo que o total das amostras analisadas estava dentro dos limites da curva.

A umidade do solo na camada de $0-5 \mathrm{~cm}$ foi estimada em cada uma das avaliações dos fluxos de gases por meio do método gravimétrico. Os valores de teor gravimétrico de água $\theta_{\mathrm{g}}\left(\mathrm{g} \mathrm{g} \mathrm{g}^{-1}\right)$ foram convertidos para proporção de espaço dos poros preenchido pela água (EPPA\%), com uso da fórmula: EPPA\% $=\left(100 \theta_{\mathrm{g}} \mathrm{d}\right) /$ [1-(d/dp)], em que d é a densidade do solo e dp é a densidade da partícula, expressas em $\mathrm{g} \mathrm{cm}^{-3}$ (Linn \& Doran, 1984).

Na determinação da densidade do solo, coletaram-se quatro amostras em cada parcela, com cilindros de $100 \mathrm{~cm}^{3}$, na profundidade de $0-5 \mathrm{~cm}$. As análises foram efetuadas no laboratório de Física e Mineralogia de Solos da Embrapa Cerrados. O valor de densidade da partícula utilizado para o cálculo do EPPA foi de $2,65 \mathrm{~g} \mathrm{~cm}^{-3}$.

Os dados foram submetidos à análise estatística de parcelas subdivididas com medidas repetidas no tempo (Vivaldi, 1999), com uso do SAS (SAS Institute, 2000). Testou-se a condição (H-F) para determinar a necessidade de corrigir ou não os graus de liberdade relativos ao tempo e à sua interação com os tratamentos, além do erro resultante desse fator. A partir da análise de variância, testaram-se os efeitos dos tipos de preparo do solo (parcela) e do período após a aplicação do fertilizante (subparcela). Os tratamentos e as interações significativas foram submetidos ao teste de Tukey, a 5\% de probabilidade.

\section{Resultados e Discussão}

Não houve diferenças significativas entre os parâmetros avaliados em relação ao preparo convencional do solo e o plantio direto. Os resultados referentes aos dois tipos de manejo do solo são apresentados em valores de médias (Tabela 2).

A densidade do solo não variou entre o preparo convencional e o plantio direto (Tabela 1), possivelmente pelo curto tempo de implantação desse sistema agrícola (três anos). Essa é uma indicação de que as propriedades físicas do solo, sob os dois métodos de preparo, não se diferenciaram para resultar em emissões de óxidos de nitrogênio ( $\mathrm{NO}$ e $\mathrm{N}_{2} \mathrm{O}$ ) significativamente diferentes, sob o preparo convencional e o plantio direto (Cárdenas et al., 1993; Sanhueza et al., 1994).

A decomposição acelerada do material vegetal das leguminosas (soja e mucuna-preta) também não favo- receu o acúmulo de resíduos e a formação de uma cobertura relevante nas parcelas sob plantio direto. Assim, as emissões de NO do solo cultivado com milho sob plantio direto não foram minimizadas pela menor exposição e maior proteção proporcionada pelas plantas de cobertura, conforme Civerolo \& Dickerson (1998).

Emissões significativamente maiores de NO (5,4 e $4,8 \mathrm{ng} \mathrm{cm}^{-2} \mathrm{~h}^{-1}$ de $\mathrm{N} ; \mathrm{p}<0,0001$ ) foram obtidas após 15 minutos (início da coleta) e três dias depois da aplicação de uréia em cobertura, respectivamente. Um dia depois da fertilização, o fluxo médio de NO foi significativamente mais baixo. Cinco dias após a aplicação do nitrogênio, o fluxo de NO decresceu novamente, com valor médio de $1,2 \mathrm{ng} \mathrm{cm}^{-2} \mathrm{~h}^{-1}$ (Figura 1), comparável ao obtido sob condição de Cerrado natural (Pinto et al., 2002).

O fluxo mais elevado de NO determinado imediatamente depois da aplicação do nitrogênio pode ser explicado pela rápida ação de ureases e pelo aumento do substrato $\left(\mathrm{NH}_{4}^{+}\right)$para nitrificação, conforme observado por Paul et al. (1993), sob condições de incubação aeróbica. O teor médio de $\mathrm{N}-\mathrm{NH}_{4}{ }^{+}$foi de $667 \mathrm{mg} \mathrm{kg}^{-1}$ de solo na primeira avaliação (Tabela 2). Skiba et al. (1997) atribuíram a magnitude da emissão de NO do solo às taxas de nitrificação e desnitrificação e às propriedades de difusão do solo.

A elevada emissão inicial de NO, possivelmente, não foi influenciada pelo nitrogênio aplicado na adubação de plantio (30 dias antes), o qual deve ter sido absorvido, em parte, pelas raízes do milho, sofrido transporte por lixiviação e, principalmente, perdas por volatilização, logo após sua aplicação (Sousa \& Lobato, 2004).

Deve-se considerar, ainda, que, no período entre a semeadura do milho (10 de novembro) e a fertilização

Tabela 2. Valores médios de $\mathrm{N}$ do $\mathrm{NO}$, do $\mathrm{NH}_{4}{ }^{+}$e do $\mathrm{NO}_{3}{ }^{-}$, teor gravimétrico de água (TG), espaço dos poros preenchido pela água (EPPA), temperatura do solo (TS) a 2,5 e 5,0 cm de profundidade, determinados entre o dia da aplicação de $\mathrm{N}$ na forma de uréia até o quinto dia depois, em um Latossolo Vermelho argiloso distrófico cultivado com milho ${ }^{(1)}$.

\begin{tabular}{|c|c|c|c|c|c|c|c|}
\hline \multirow[t]{2}{*}{ Período } & \multirow{2}{*}{$\begin{array}{c}\mathrm{N}-\mathrm{NO} \\
\left(\mathrm{ng} \mathrm{cm}^{-2} \mathrm{~h}^{-1}\right)\end{array}$} & \multirow{2}{*}{$\begin{array}{l}\mathrm{N}^{\mathrm{N}} \mathrm{NH}_{4}^{+} \\
---(\mathrm{mg} \mathrm{kg}\end{array}$} & \multirow{2}{*}{$\begin{array}{l}\mathrm{N}-\mathrm{NO}_{3}^{-} \\
\mathrm{g}^{-1} \text { ) ---- }\end{array}$} & \multirow{2}{*}{$\begin{array}{c}\mathrm{TG} \\
\left(\mathrm{g} \mathrm{g}^{-1}\right)\end{array}$} & \multirow{2}{*}{$\begin{array}{c}\text { EPPA } \\
(\%)\end{array}$} & \multicolumn{2}{|c|}{$\operatorname{TS}\left({ }^{\circ} \mathrm{C}\right)$} \\
\hline & & & & & & $2,5 \mathrm{~cm}$ & $5,0 \mathrm{~cm}$ \\
\hline 0 & $5,4 \mathrm{a}$ & $667 \mathrm{ab}$ & $61 \mathrm{a}$ & $33 a$ & $61 \mathrm{a}$ & $35,2 \mathrm{a}$ & $32,8 \mathrm{a}$ \\
\hline 1 & $1,9 \mathrm{~b}$ & $616 a b$ & $61 \mathrm{a}$ & $31 \mathrm{a}$ & $58 \mathrm{a}$ & $31,0 \mathrm{~b}$ & $29,7 \mathrm{~b}$ \\
\hline 2 & $4,8 \mathrm{a}$ & $786 a$ & $47 a$ & $26 \mathrm{~b}$ & $48 b$ & $24,5 \mathrm{c}$ & $24,4 \mathrm{~d}$ \\
\hline 3 & $-(2)$ & $677 \mathrm{ab}$ & $52 \mathrm{a}$ & $20 \mathrm{c}$ & $36 c$ & $29,4 \mathrm{~b}$ & $27,5 \mathrm{bc}$ \\
\hline 5 & $1,2 \mathrm{~b}$ & $461 \mathrm{~b}$ & $62 \mathrm{a}$ & $17 \mathrm{c}$ & $34 \mathrm{c}$ & $25,3 \mathrm{c}$ & $25,4 \mathrm{~cd}$ \\
\hline
\end{tabular}

(1)Médias seguidas de mesma letra na coluna não diferem pelo teste de Tukey, a 5\% de probabilidade. (2)Não foi coletada. 
em cobertura (10 de dezembro), a precipitação pluvial acumulada foi de $103 \mathrm{~mm}$, e que $41 \mathrm{~mm}$ ocorreram nos dez dias anteriores à fertilização de cobertura. Assim, a quantidade de precipitação entre a fertilização de plantio e a de cobertura, provavelmente, impediu o acúmulo de $\mathrm{NO}_{2}{ }^{-}$, que poderia gerar fluxos de $\mathrm{NO}$ por quimionitrificação (Davidson et al., 1993; Skiba et al., 1997), considerando o valor de $\mathrm{pH}$ desse solo (Tabela 1). Baixas emissões de $\mathrm{N}_{2} \mathrm{O}$ também indicam que a nitrificação deve ter sido o processo dominante para a elevada emissão de NO, imediatamente após a aplicação de uréia (Davidson et al., 2000).

O valor médio de EPPA (61\%) e a temperatura do solo $\left(35,2^{\circ} \mathrm{C}\right.$ a $2,5 \mathrm{~cm}$ e $32,8^{\circ} \mathrm{C}$ a $\left.5 \mathrm{~cm}\right)$, significativamente mais altos do que nos demais períodos (Tabela 2), podem ter contribuído para a elevada emissão de NO medida logo depois da fertilização com nitrogênio. O intervalo de EPPA entre 20 e 60\% é considerado ideal para a produção de NO (Davidson et al., 2000; Potter et al., 2001). Skiba et al. (1994) encontraram correlações dos fluxos de NO com a temperatura do solo, que juntamente ao $\mathrm{NO}_{3}{ }^{-}$, explicaram $60 \%$ das emissões deste gás em área fertilizada. McKenney \& Drury (1997) relacionaram as variações diurnas dos fluxos de NO com a temperatura na superfície do solo. Yamulki et al. (1995) observaram que as medidas de fluxos de NO em área fertilizada acompanharam as variações sazonais de $\mathrm{NH}_{4}{ }^{+}, \mathrm{NO}_{3}{ }^{-}$e umidade do solo. As concentrações de nitrato não apresentaram diferenças significativas ao longo do experimento (Tabela 2).

Apesar de os resultados indicarem essa relação entre fluxo de $\mathrm{NO}$ e disponibilidade de água e de $\mathrm{NH}_{4}{ }^{+}$no

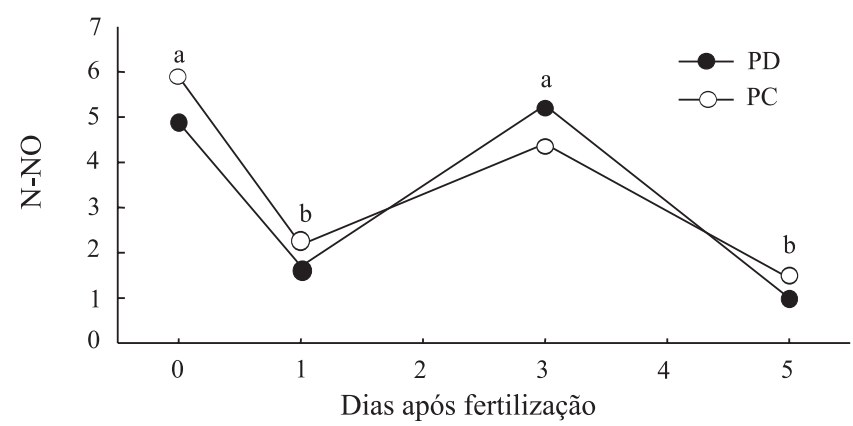

Figura 1. Fluxo de $\mathrm{N}$ do $\mathrm{NO}\left(\mathrm{ng} \mathrm{cm}^{-2} \mathrm{~h}^{-1}\right.$ ), entre o dia da aplicação de $\mathrm{N}$ (uréia) e o quinto dia depois, nos sistemas plantio convencional (PC) e plantio direto (PD), em um Latossolo Vermelho argiloso distrófico cultivado com milho. Médias com a mesma letra não diferem entre si pelo teste de Tukey, a 5\% de probabilidade. solo, o incremento da emissão de NO após a fertilização foi de curta duração. Houve redução significativa na emissão de NO já no primeiro dia após aplicação de uréia em cobertura (de 5,1 para 1,9 $\mathrm{ng} \mathrm{cm}^{-2} \mathrm{~h}^{-1}$ de N). A manutenção das condições de elevada umidade do solo (Tabela 2) pode ter contribuído para reduzir a difusão de NO pelos poros (Cárdenas et al., 1993; Potter et al., 2001). Davidson et al. (2000) indicaram que o valor de EPPA em torno de $60 \%$ é o limite entre os processos microbianos limitados por água ou pela aeração.

A concentração de $\mathrm{NH}_{4}{ }^{+}$também foi mantida estável no período entre as duas primeiras avaliações. Entretanto, houve uma redução significativa da temperatura a $2,5 \mathrm{~cm}\left(35,2\right.$ para $\left.31,0^{\circ} \mathrm{C}\right)$ e $5,0 \mathrm{~cm}(32,8$ para $29,7^{\circ} \mathrm{C}$ ), embora esses valores medidos no dia seguinte à aplicação de nitrogênio ainda sejam elevados (Tabela 2).

O elevado fluxo de NO (Figura 1), observado no terceiro dia depois da aplicação de uréia, pode ter ocorrido em conseqüência da disponibilidade ainda alta de $\mathrm{NH}_{4}{ }^{+}$ e da redução do EPPA a 36\% (Tabela 2), permitindo maior difusão deste gás no solo (Davidson et al., 2000; Potter et al., 2001).

A menor concentração média de $\mathrm{NH}_{4}{ }^{+}$no solo foi alcançada no quinto dia depois da aplicação de uréia em cobertura (Tabela 2), quando também se observou o decréscimo do fluxo de NO, atingindo valor médio de $1,2 \mathrm{ng} \mathrm{cm}^{-2} \mathrm{~h}^{-1}$ de N (Figura 1), semelhante ao relatado sob Cerrado natural (Pinto et al., 2002).

Erickson et al. (2001) sugerem que as plantas e os microrganismos heterotróficos funcionam como drenos, necessitando saturarem-se com nitrogênio para, em seguida, disponibilizá-lo para reações de nitrificação.

A maioria das medidas de $\mathrm{N}_{2} \mathrm{O}$ realizadas no período imediatamente e até cinco dias depois da fertilização em cobertura apresentaram fluxos inferiores ao limite de detecção $\left(0,6 \mathrm{ng} \mathrm{cm}^{-2} \mathrm{~h}^{-1}\right.$ de $\left.\mathrm{N}\right)$. Resultados semelhantes foram obtidos para solos de Cerrado, sob boas condições de aeração, como o Latossolo Vermelho (Pinto et al., 2002; Varella et al., 2004). A variabilidade obtida quanto aos dados de $\mathrm{N}_{2} \mathrm{O}$ é bastante acentuada, com desvios em relação aos valores médios. Considerando os valores obtidos imediatamente após a aplicação da uréia sob o plantio direto $\left(-0,1 \mathrm{ng} \mathrm{cm}^{-2} \mathrm{~h}^{-1}\right.$ de $\left.\mathrm{N}\right)$ e o plantio convencional (-0,5 $\mathrm{ng} \mathrm{cm}^{-2} \mathrm{~h}^{-1}$ de N), os desvios foram de 1,3 e de 2,7 , respectivamente.

Williams et al. (1992) identificaram a cultura de milho como a maior fonte biogênica de NO nos Estados Unidos - responsável por $40 \%$ das emissões -, apesar 
de essa cultura ocupar somente 4\%, em área. Altas emissões estariam relacionadas ao elevado requerimento de fertilizantes nitrogenados para sua produtividade. Sanhueza et al. (1994) não observaram incrementos nas emissões de NO em solo de savanas por meio da aplicação exclusiva de $\mathrm{NH}_{4}{ }^{+}$, porém a aplicação simultânea de $\mathrm{NO}_{3}{ }^{-}$e de $\mathrm{NH}_{4}{ }^{+}$aumentou os fluxos desse gás. Skiba et al. (1997) sugerem o uso de plantas de cobertura no sistema agrícola como uma prática eficiente para reduzir as emissões de NO nas culturas subseqüentes.

No Brasil, não há estimativas das emissões agrícolas de NO baseadas em medições de campo. O entendimento da dinâmica entre práticas de cultivo fórmula do fertilizante, tipo de preparo do solo, uso de plantas de cobertura, irrigação, entre outros - e fluxos de NO - incluindo a freqüência de altos fluxos - é fundamental para a proposição de estratégias de mitigação da emissão de gases de efeito estufa.

\section{Conclusões}

1. As condições de oferta de água e de nitrogênio, além da temperatura do solo, proporcionam valores elevados de emissão de NO imediatamente após e no terceiro dia depois da aplicação de uréia em cobertura; depois de cinco dias da fertilização, a emissão de NO é semelhante à de solo sob vegetação nativa de cerrado.

2. A adequada aeração do solo e a disponibilidade de nitrogênio na forma amoniacal favorecem a emissão de NO para a atmosfera, mas não a de $\mathrm{N}_{2} \mathrm{O}$.

3. A dinâmica dos fluxos de NO, no curto período após a fertilização em cobertura, não é influenciada pelo tipo de preparo do solo (preparo convencional e plantio direto).

\section{Agradecimentos}

À Embrapa Cerrados, pelo apoio logístico; a Laura T. Viana, pelas análises laboratoriais; à NASA (LBA ND-07) e United States Environmental Protection Agency, pelo financiamento parcial deste trabalho.

\section{Referências}

CÁRDENAS, L.; RONDÓN, A.; JOHANSSON, C.; SANHUEZA, E. Effects of soil-moisture, temperature, and inorganic nitrogen on nitric-oxide emissions from acidic tropical savanna soils. Journal of Geophysical Research, v.98, p.14783-14790, 1993.
CIVEROLO, K.L.; DICKERSON, R.R. Nitric oxide soil emissions from tilled and untilled cornfields. Agricultural and Forest Meteorology, v.90, p.307-311, 1998.

DAVIDSON, E.A.; KELLER, M.; ERICKSON, H.E.; VERCHOT, L.V.; VELDKAMP, E. Testing a conceptual model of soil emissions of nitrous and nitric oxides. BioScience, v.50, p.667-680, 2000.

DAVIDSON, E.A.; MATSON, P.A.; BROOKS, P.D. Nitrous oxide emission controls and inorganic nitrogen dynamics in fertilized tropical agricultural soils. Soil Science Society of America Journal, v.60, p.1145-1152, 1996.

DAVIDSON, E.A.; MATSON, P.A.; VITOUSEK, P.M.; RILEY, R.; DUNKIN, K.; GARCÍA-MÉNDEZ, G.; MAASS, J.M. Processes regulating soil emissions of $\mathrm{NO}$ and $\mathrm{N}_{2} \mathrm{O}$ in a seasonally dry tropical forest. Ecology, v.74, p.130-139, 1993.

ERICKSON, H.E.; KELLER, M.; DAVIDSON, E.A. Nitrogen oxide fluxes and nitrogen cycling during postagricultural succession and forest fertilization in the humid tropics. Ecosystems, v.4, p.67-84, 2001.

GREWELING, T.; PEECH, M. Chemical soil tests. Ithaca, NY: Cornell University Agricultural Experiment Station, New York State College of Agriculture, 1960. 54p.

LINN, D.M.; DORAN, J.W. Effect of water-filled pore-space on carbon-dioxide and nitrous-oxide production in tilled and nontilled soils. Soil Science Society of America Journal, v.48, p.12671272, 1984.

McKENNEY, D.J.; DRURY, C.F. Nitric oxide production in agricultural soils. Global Change Biology, v.3, p.317-326, 1997.

MATSON, P.A.; McDOWELL, W.H.; TOWNSEND, A.R.; VITOUSEK, P.M. The globalization of N deposition: ecosystem consequences in tropical environments. Biogeochemistry, v.46, p.6783, 1999.

MEIER, M. Nitratbestimmung in Boden-proben (N-min-Method). LaborPraxis, v.4, p.244-247, 1991.

NOBRE, A.D. Nitrous oxide emissions from tropical soils. 1994. 141p. Thesis (Ph.D.) - University of New Hampshire, Durham.

PAUL, J.W.; BEAUCHAMP, E.G.; ZHANG, X. Nitrous and nitricoxide emissions during nitrification and denitrification from manureamended soil in the laboratory. Canadian Journal of Soil Science, v.73, p.539-553, 1993.

PINTO, A. de S.; BUSTAMANTE, M.M.C.; KISSELLE, K.; BURKE, R.; ZEPP, R.; VIANA, L.T.; VARELLA, R.F.; MOLINA, M. Soil emissions of $\mathrm{N}_{2} \mathrm{O}$, $\mathrm{NO}$ and $\mathrm{CO}_{2}$ in Brazilian Savannas: effects of vegetation type, seasonality, and prescribed fires. Journal of Geophysical Research, v.107, p.8089-8095, 2002.

POTTER, C.; DAVIDSON, E.; NEPSTAD, D.; CARVALHO, C.R. de. Ecosystem modeling and dynamic effects of deforestation on trace gas fluxes in Amazon tropical forests. Forest Ecology and Management, v.152, p.97-117, 2001. 
SAMINÊZ, T.C. de O. Efeito do sistema de cultivo, tensão da água, biomassa microbiana e temperatura do solo nos fluxos de $\mathbf{C H}_{4}$ e $\mathbf{N}_{2} \mathbf{O}$ em solos de cerrados. 1999. 99p. Dissertação (Mestrado) - Universidade de Brasília, Brasília.

SANHUEZA, E.; CÁRDENAS, L.; DONOSO, L.; SANTANA, M. Effect of plowing on $\mathrm{CO}_{2}, \mathrm{CO}, \mathrm{CH}_{4}, \mathrm{~N}_{2} \mathrm{O}$, and $\mathrm{NO}$ fluxes from tropical savanna soils. Journal of Geophysical Research, v.99, p.16429-16434, 1994.

SAS INSTITUTE (Cary, Estados Unidos). SAS/STAT: user's guide, version 8.1. Cary, 2000. v.1. 943p.

SKIBA, U.M.; FOWLER, D.; SMITH, K.A. Emissions of NO and $\mathrm{N}_{2} \mathrm{O}$ from soils. In: HAM, J. van; JANSSEN, L.H.J.M.; SWART, R.J. (Ed.). Non- $\mathrm{CO}_{2}$ greenhouse gases: why and how to control? Dordrecht: Kluwer Academic, 1994. p.153-158.

SKIBA, U.M.; FOWLER, D.; SMITH, K.A. Nitric oxide emissions from agricultural soils in temperate and tropical climates: sources, controls and mitigation options. Nutrient Cycling in Agroecosystems, v.48, p.139-153, 1997.

SKIBA, U.M.; SHEPPARD, L.J.; MacDONALD, J.; FOWLER, D. Some key environmental variables controlling nitrous oxide emissions from agricultural and semi-natural soils in Scotland. Atmospheric Environment, v.32, p.3311-3320, 1998.

SLEMR, F.; SEILER, W. Field-study of environmental variables controlling the NO emissions from soil and the NO compensation point. Journal of Geophysical Research, v.96, p.13017-13031, 1991.

SOUSA, D.M.G. de; LOBATO, E. Adubação com nitrogênio. In: SOUSA, D.M.G. de; LOBATO, E. (Ed.). Cerrado: correção do solo e adubação. Brasília: Embrapa Informação Tecnológica, 2004. p.129145.

VARELLA, R.F.; BUSTAMANTE, M.M.C.; PINTO, A.S.; KISSELLE, K.W.; SANTOS, R.V.; BURKE, R.A.; ZEPP, R.G.; VIANA, L.T. Soil fluxes of $\mathrm{CO}_{2}, \mathrm{CO}, \mathrm{NO}$, and $\mathrm{N}_{2} \mathrm{O}$ from an old pasture and from native savanna in Brazil. Ecological Applications, v.14, p.221-231, 2004.

VELDKAMP, E.; KELLER, M.; NUÑEZ, M. Effects of pasture management on $\mathrm{N}_{2} \mathrm{O}$ and $\mathrm{NO}$ emissions from soils in the humid tropics of Costa Rica. Global Biogeochemical Cycles, v.12, p.7179, 1998.

VERCHOT, L.V.; DAVIDSON, E.A.; CATTÂNIO, J.H.; ACKERMAN, I.L.; ERICKSON, H.E.; KELLER, M. Land use change and biogeochemical controls of nitrogen oxide emissions from soils in eastern Amazonia. Global Biogeochemical Cycles, v.13, p.31-46, 1999.

VIVALDI, L.J. Análise de experimentos com dados repetidos ao longo do tempo ou espaço. Planaltina: Embrapa-CPAC, 1999. 52p. (Embrapa-CPAC. Documentos, 8).

VOS, G.J.M.; BERGEVOET, I.M.J.; VEDY, J.C.; NEYROUD, J.A. The fate of spring applied fertilizer- $\mathrm{N}$ during the autumn-winter period: comparison between winter-fallow and green manure cropped soil. Plant and Soil, v.160, p.201-213, 1994.

WILliAMS, E.J.; GUENTHER, A.; FEHSENFELD, F.C. An inventory of nitric-oxide emissions from soils in the United States. Journal of Geophysical Research, v.97, p.7511-7519, 1992.

YAMULKI, S.; GOULDING, K.W.T.; WEBSTER, C.P.; HARRISON, R.M. Studies on $\mathrm{NO}$ and $\mathrm{N}_{2} \mathrm{O}$ fluxes from a wheat field. Atmospheric Environment, v.29, p.1627-1635, 1995.

Recebido em 3 de março de 2005 e aprovado em 30 de setembro de 2005 